\title{
Robust indicator-based algorithm for interactive evolutionary multiple objective optimization e-Appendix (supplementary material)
}

\author{
Michał K. Tomczyk, Miłosz Kadziński \\ Institute of Computing Science, Poznań University of Technology \\ Poznań, Poland \\ \{michal.tomczyk,milosz.kadzinski\}@cs.put.poznan.pl
}

\section{ACM Reference Format:}

Michał K. Tomczyk, Miłosz Kadziński. 2019. Robust indicator-based algorithm for interactive evolutionary multiple objective optimization e-Appendix (supplementary material) . In Proceedings of the Genetic and Evolutionary Computation Conference 2019 (GECCO '19). ACM, New York, NY, USA, 5 pages.

In the e-Appendix we discuss the results of additional experiments which were not included in the main paper. Specifically, we:

- we discuss how we derived the most relevant Pareto-optimal solutions for different artificial Decision Makers (DMs) (Section 1),

- we analyze how many times the extreme points changed throughout the evolutionary search, triggering the Monte Carlo simulation for finding compatible model instances (Section 2).

- we compare two variants of IEMO/I assuming either estimated or fixed extreme points (Section 3),

\section{FINDING PARETO-OPTIMAL SOLUTIONS FOR DIFFERENT ARTIFICIAL DMs}

In this section, we discuss how we derived the most relevant Paretooptimal solutions for different artificial DMs. Let us remind that in the main paper:

(1) We considered WFG1-9 [1] benchmark problems whose objective functions employ dissimilar domains. Specifically, WFG problems multiply the $i^{\text {th }}$ objective value $f_{i}$ by $2(i+1)$.

(2) To model the DM's preferences, we used a weighted Chebyshev function $f_{C F}^{w^{D M}}$ which normalizes the objective values according to the objective ranges of WFG benchmarks. Precisely, each value on the $i^{t h}$ objective is divided by $2(i+1)$.

In this regard, to simplify the notation and avoid redundant transformations (i.e., multiplying and dividing by the same number), in this appendix we simply avoid $2(i+1)$ factor when computing the optimal solutions.

To find a Pareto-optimal solution minimizing $f_{C F}^{w^{D M}}$, one can construct a vector $v_{\theta}^{w^{D M}}=\left[\theta / w_{1}^{D M}, \ldots \theta / w_{M}^{D M}\right]$, where $\theta>0$

Permission to make digital or hard copies of all or part of this work for personal or classroom use is granted without fee provided that copies are not made or distributed for profit or commercial advantage and that copies bear this notice and the full citation on the first page. Copyrights for components of this work owned by others than ACM must be honored. Abstracting with credit is permitted. To copy otherwise, or republish, to post on servers or to redistribute to lists, requires prior specific permission and/or a fee. Request permissions from permissions@acm.org.

GECCO '19, fuly 13-17, 2019, Prague, Czech Republic

(C) 2019 Association for Computing Machinery. controls its length, and find its intersection with the Pareto-front (PF) [2]. This procedure can be applied to WFG1 and WFG3-9 benchmarks having continuous PFs. In turn, WFG2 has a disconnected $\mathrm{PF}$ and this procedure cannot be used as $v_{\theta}^{w^{D M}}$ may simply not intersect with the PF. For this reason, we applied a heuristic method to find the optimal solutions for WFG2.

Overall, we consider the following scenarios, which are ordered from the easiest to the most difficult:

(1) WFG3 with a flat Pareto-front (Section 1.1.1);

(2) WFG4-9 with concave Pareto-fronts (Section 1.1.2);

(3) WFG1 with a mixed Pareto-front (Section 1.1.3);

(4) cWFG4-5 and cWFG6-9 with convex Pareto-fronts (Section 1.1.4);

(5) WFG2 with a disconnected Pareto-front (Section 1.1.5).

Furthermore, in Section 5.3 of the main paper, we consider the convex counterparts of WFG4-5, denoted by cWFG4-5, and an artificial DM modelled with a weighted sum $f_{W S}^{w^{D M}}$. Such a scenario is handled in Section 1.2.

\subsection{Optimal solutions for a simulated DM modelled with the Weighted Chebyshev function}

1.1.1 Optimal solutions for WFG3. WFG3 has a flat PF (see Figure 1). Let us remind that, to simplify the notation, we do not rescale

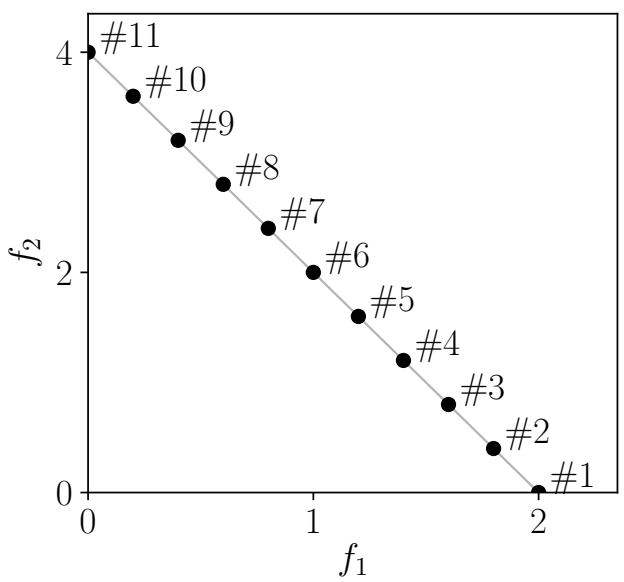

Figure 1: The PF of WFG3 (grey line) and 11 solutions minimizing $f_{C F}^{w^{D M, i}}$ (black dots). The \#i solution corresponds to $w^{D M, i}=[(i-1) / 10,1-(i-1) / 10]$ objective weight vector. The PF is marked with a grey line. 
the PFs of WFG problems. Hence, the PF of WFG3 can be modelled with the following non-parametric equation:

$$
f_{1}+\ldots+f_{M}=1 \text {. }
$$

Then, one can substitute $f_{i}$ with $\theta / w_{i}^{D M}$ and find the scaling factor $\theta$ :

$$
\theta=1 / \sum_{i=1}^{M}\left(1 / w_{i}^{D M}\right)
$$

Note that, when some $w_{i}^{D M}$ is equal to 0 , we substitute it with an arbitrarily small value of $10^{-9}$ to avoid dividing by 0 . When $\theta$ is already known, one may compute a Pareto-optimal solution minimizing $f_{C F}^{w^{D M}}$. In Figure 1, we illustrate the derived intersection points representing the optimal solutions for $M=2$ and 11 uniformly distributed objective weight vectors, i.e., $w^{D M_{1}}=[0.0,1.0]$, $w^{D M_{2}}=[0.1,0.9], \ldots, w^{D M_{11}}=[1.0,0.0]$.

1.1.2 Optimal solutions for WFG4-9. WFG4-9 have concave spherical PFs (see Figure 2). Their PFs can be modelled with a nonparametric equation of the following form:

$$
f_{1}^{2}+\ldots f_{M}^{2}=1 \text {. }
$$

Analogously to WFG3, one may use the following transformation to find a scaling factor $\theta$ and thus the optimal solutions:

$$
\theta^{2}=1 / \sum_{i=1}^{M}\left[1 /\left(w_{i}^{D M}\right)^{2}\right] \text {. }
$$

Equation (4) has two solutions. However, we assumed that $\theta>0$, and thus neglected the solution having negative objective values. In Figure 2, we illustrate the example optimal solutions for $M=2$ and 11 uniformly distributed weight vectors.

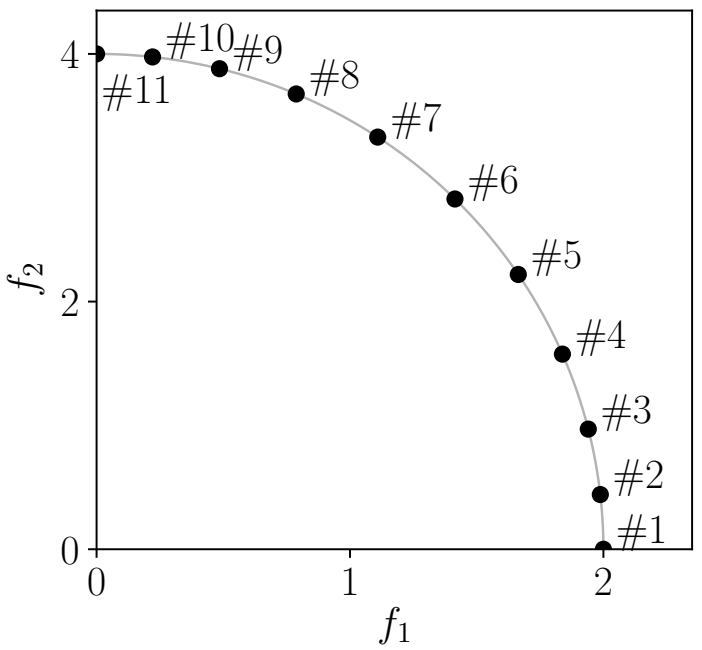

Figure 2: The PFs of WFG4-9 (grey line) and 11 solutions minimizing $f_{C F}^{w^{D M, i}}$ (black dots). The \#i solution corresponds to $w^{D M, i}=[(i-1) / 10,1-(i-1) / 10]$ objective weight vector.
1.1.3 Optimal solutions for WFG1. WFG1 has a more complex shape of the PFs than for the remaining benchmark problems. Specifically, it has a mixed PF consisting of convex and concave regions (see Figure 3). Since its non-parametric equation is not known, we used its parametric equivalent defined as follows:

$$
\begin{aligned}
f_{i=1} & =\prod_{j=1}^{M-1}\left(1-\cos \left(x_{j} \pi / 2\right)\right) \\
f_{i \in\{2, \ldots, M-1\}} & =\left(\prod_{j=1}^{M-i}\left(1-\cos \left(x_{j} \pi / 2\right)\right)\right)\left(1-\sin \left(x_{M-i+1} \pi / 2\right)\right) \\
f_{i=M} & =1-x_{1}-\frac{\cos \left(10 \pi x_{1}+\pi / 2\right)}{10 \pi}
\end{aligned}
$$

where $x$ is the problem's internal vector of $M-1$ parameters $\left(x_{j} \in\right.$ $[0,1])$.

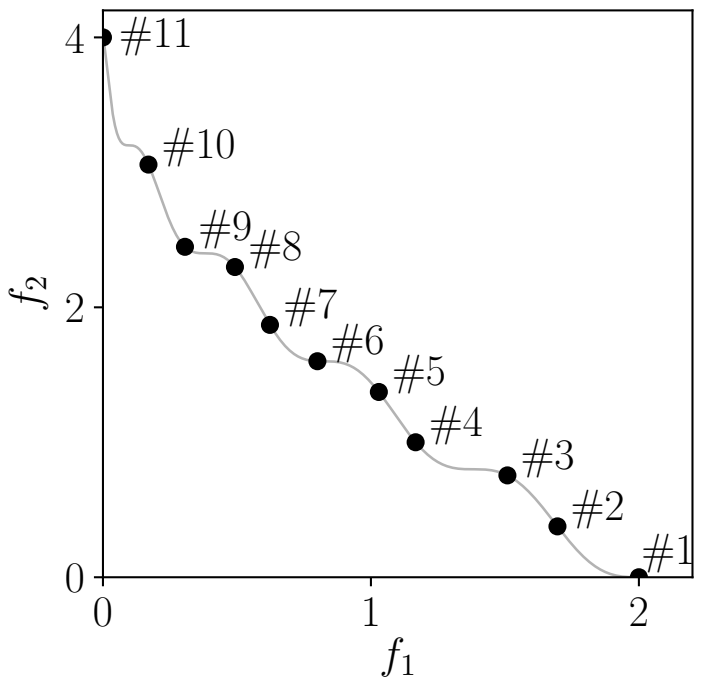

Figure 3: The PFs of WFG1 (grey line) and 11 solutions minimizing $f_{C F}^{w^{D M, i}}$ (black dots). The \#i solution corresponds to $w^{D M, i}=[(i-1) / 10,1-(i-1) / 10]$ objective weight vector.

Firstly, we again assumed that $f_{i}=\theta / w_{i}^{D M}$. Then, we iteratively computed the values of $x_{k}$ for $k=M-1, \ldots, 1$. Having computed the parameter vector $x$, we constructed the optimal solution.

Computing $x_{k}$ for $k=M-1$ and $M \geq 3$. We considered the following set of equations:

$$
\left\{\begin{array}{l}
\theta / w_{1}^{D M}=\prod_{j=1}^{M-1}\left(1-\cos \left(x_{j} \pi / 2\right)\right) \\
\theta / w_{2}^{D M}=\left(\prod_{j=1}^{M-2}\left(1-\cos \left(x_{j} \pi / 2\right)\right)\right)\left(1-\sin \left(x_{M-1} \pi / 2\right)\right) .
\end{array}\right.
$$

Consequently:

$$
w_{2}^{D M} / w_{1}^{D M}=\frac{1-\cos \left(x_{M-1} \pi / 2\right)}{1-\sin \left(x_{M-1} \pi / 2\right)} .
$$


Equation (10) is difficult to solve. However, note that $1-\cos \left(x_{M-1} \pi / 2\right)$ monotonically increases while $1-\sin \left(x_{M-1} \pi / 2\right)$ decreases with the increase of $x_{M-1}$. Hence, the whole fraction is an increasing function and thus the underlying equation can be easily solved with some numerical method guaranteeing fast convergence toward optimum. In our study, we firstly modelled $x_{M-1}$ variable in the following way:

$$
x_{M-1}=\frac{1}{2^{1}} B_{1}+\frac{1}{2^{2}} B_{2}+\frac{1}{2^{3}} B_{2} \ldots,
$$

where $B_{l} \in\{0,1\}$. Hence, $x_{M-1}$ was initially set to 0 . Then, we iteratively, i.e., for $l=1,2,3, \ldots$, checked if $x_{M-1}$ could be increased by $1 / 2^{l}$. Precisely, $1 / 2^{l}$ was added to $x_{M-1}$ if the right side of Equation (10) - evaluated in terms of such updated $x_{M-1}-$ did not exceed $w_{2}^{D M} / w_{1}^{D M}$. The difference quantified the error rate used as a stopping criterion. Specifically, the procedure stopped when the error rate was lesser than $10^{-9}$.

Computing $x_{k}$ for $k=M-2, \ldots, 2$ and $M \geq 4$. We formulated the following set of equations:

$$
\left\{\begin{array}{l}
\theta / w_{M-k}^{D M}=\left(\prod_{j=1}^{k}\left(1-\cos \left(x_{j} \pi / 2\right)\right)\right)\left(1-\sin \left(x_{k+1} \pi / 2\right)\right), \\
\theta / w_{M-k+1}^{D M}=\left(\prod_{j=1}^{k-1}\left(1-\cos \left(x_{j} \pi / 2\right)\right)\right)\left(1-\sin \left(x_{k} \pi / 2\right)\right),
\end{array}\right.
$$

which can be transformed to:

$$
\frac{w_{M-k+1}^{D M}}{w_{M-k}^{D M}\left(1-\sin \left(x_{k+1} \pi / 2\right)\right)}=\frac{1-\cos \left(x_{k} \pi / 2\right)}{1-\sin \left(x_{k} \pi / 2\right)} .
$$

As with Equation 10, we used the numerical method described above to find the value of $x_{k}$.

Computing $x_{k}$ for $k=1$. When $M \geq 3$, we considered the following set of constraints:

$$
\left\{\begin{array}{l}
\theta / w_{M-1}^{D M}=\left(1-\cos \left(x_{1} \pi / 2\right)\right)\left(1-\sin \left(x_{2} \pi / 2\right)\right), \\
\theta / w_{M}^{D M}=1-x_{1}-\frac{\cos \left(10 \pi x_{1}+\pi / 2\right)}{10 \pi}
\end{array}\right.
$$

which gives:

$$
\frac{w_{M}^{D M}}{w_{M-1}^{D M}\left(1-\sin \left(x_{2} \pi / 2\right)\right)}=\frac{\left(1-\cos \left(x_{1} \pi / 2\right)\right)}{1-x_{1}+\frac{\sin \left(10 \pi x_{1}\right)}{10 \pi}} .
$$

Note that when $M=2$, the factor of $\sin \left(x_{2} \pi / 2\right)$ should be removed from the left side of this equation.

Let us again prove that the right side of Equation (15) monotonically increases with the increase of $x_{1}$, and thus the previously discussed numerical method can be applied to solve this equation. Firstly, as with Equation (10), the nominator increases with the increase of $x_{1}$. Thus, we only need to show that the denominator is a decreasing function. For this reason, let us compute its derivative:

$$
\begin{aligned}
& {\left[1-x_{1}+\frac{\sin \left(10 \pi x_{1}\right)}{10 \pi}\right]^{\prime}=-1+\left[\sin \left(10 \pi x_{1}\right)\right]^{\prime}=} \\
& -1+\frac{1}{10 \pi} \cdot 10 \pi \cdot \cos \left(10 \pi x_{1}\right)=-1+\cos \left(10 \pi x_{1}\right) .
\end{aligned}
$$

For $x_{1} \in[0,1]$ the computed derivative is non-positive and thus the denominator of Equation (15) is a decreasing function. This proves that Equation (15) increases with the increase of $x_{1}$. For this reason, our numerical method, which guarantees convergence toward optimum, can be applied.

Finally, in Figure 3, we illustrate the example optimal solutions for $M=2$ and 11 uniformly distributed weight vectors $w^{D M}$.

1.1.4 Optimal solutions for cWFG4-5 and cWFG6-9. In the main paper we consider convex counterparts of WFG4-5 problems, denoted by cWFG4-5, when comparing IEMO/I with NEMO methods. Note that all cWFG4-9 benchmarks have the same PF. Hence, the discussion provided in this section is valid for cWFG6-9 problems as well. Following [1], we modelled the PFs of cWFG4-9 with the following objective functions:

$$
\begin{aligned}
f_{i=1} & =\prod_{j=1}^{M-1}\left(1-\cos \left(x_{j} \pi / 2\right)\right) \\
f_{i \in\{2, \ldots, M-1\}} & =\left(\prod_{j=1}^{M-i}\left(1-\cos \left(x_{j} \pi / 2\right)\right)\right)\left(1-\sin \left(x_{M-i+1} \pi / 2\right)\right) \\
f_{i=M} & =1-\sin \left(x_{1} \pi / 2\right)
\end{aligned}
$$

Note that, although WFG4-9 have concave PFs which are spherical, thus modelled cWFG4-9 have convex PFs which, in turn, are spherical only for $M=2$. Hence, we could not use a non-parametric equation as with WFG4-9 to find the optimal solutions. Instead, we used its parametric equivalent defined by Equations (18)-(20). Nonetheless, except for $i=M$, the objective functions of cWFG4-9 are defined in the same way as these of WFG1. Thus, for $k=M-1$ and $k<M-1$ we solved, respectively, Equation (10) and Equation (13) to construct the optimal solutions. In Figure 4, we illustrate the example optimal solutions for $M=2$ and 11 uniformly distributed weight vectors $w^{D M}$.

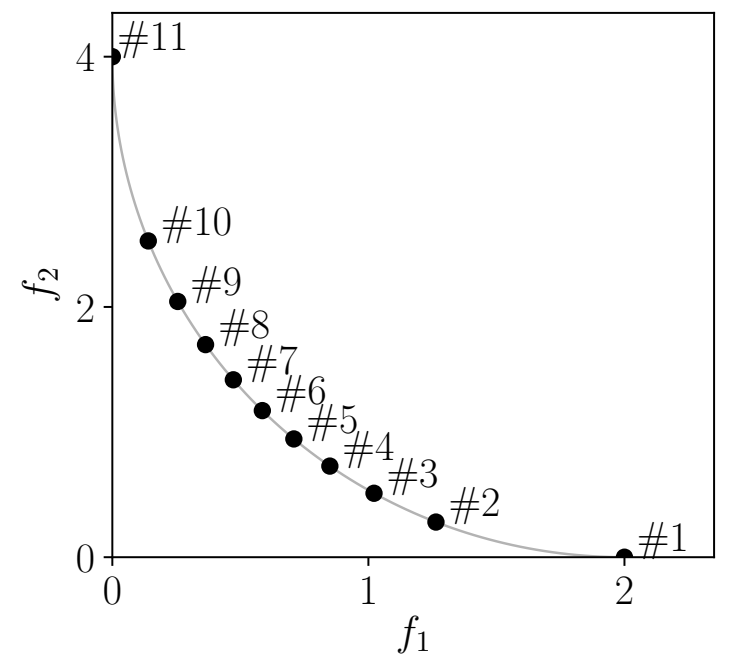

Figure 4: The PFs of cWFG4-9 (grey line) and 11 solutions minimizing $f_{C F}^{w^{D M, i}}$ (black dots). The \#i solution corresponds to $w^{D M, i}=[(i-1) / 10,1-(i-1) / 10]$ objective weight vector. 
1.1.5 Optimal solutions for WFG2. WFG2 has a disconnected PF (see Figure 5) modelled in the following way:

$$
\begin{aligned}
f_{i=1} & =\prod_{j=1}^{M-1}\left(1-\cos \left(x_{j} \pi / 2\right)\right) \\
f_{i \in\{2, \ldots, M-1\}} & =\left(\prod_{j=1}^{M-i}\left(1-\cos \left(x_{j} \pi / 2\right)\right)\right)\left(1-\sin \left(x_{M-i+1} \pi / 2\right)\right) \\
f_{i=M} & =1-x_{1} \cos ^{2}\left(5 x_{1} \pi\right) .
\end{aligned}
$$

Due to WFG2 having a disconnected PF, we could not use the geometrical approach to find the optimal solutions for this problem. Instead, we used a heuristic method. Specifically, we employed a non-interactive variant of ECC-MRW incorporating an a priori known preference function $f_{C F}^{w^{D M}}$ of the artificial DM. Then, we set the number of position- $(k)$ and distance-related $(l)$ parameters of $W F G 2$ to their minimal values. Precisely, $k$ was set to $M-1$ and $l$ to 2 . This gives only 6 decision variables for the most difficult scenario involving $M=5$ objectives. Such a low number of parameters made the underlying problem easy to solve. To find an optimal solution, we run the a priori variant of ECC-MRW on thus parametrized WFG2 100 times. Then, we selected a solution optimizing $f_{C F}^{w^{D M}}$ in the best way, and considered it as the optimal one. In Figure 4, we illustrate the example optimal solutions for $M=2$ and 11 uniformly distributed weight vectors $w^{D M}$.

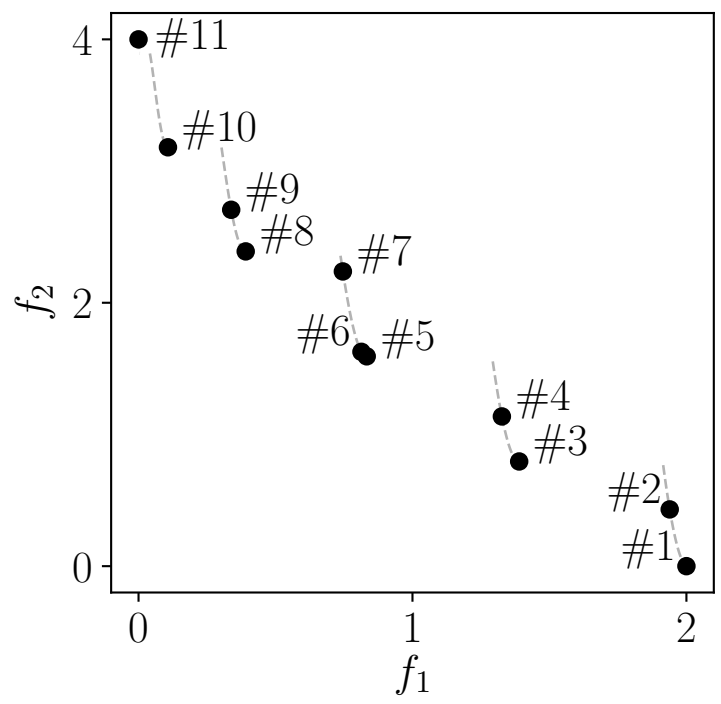

Figure 5: The PFs of WFG2 (grey line) and 11 solutions minimizing $f_{C F}^{w^{D M, i}}$ (black dots). The \#i solution corresponds to $w^{D M, i}=[(i-1) / 10,1-(i-1) / 10]$ objective weight vector.

\subsection{Optimal solutions for a simulated DM modelled with the Weighted Sum function}

In Section 5.3 of the main paper, we modelled the artificial DM with a Weighted Sum function:

$$
f_{W S}^{w^{D M}}(s)=\sum_{i=1, \ldots, M} w_{i} s_{i} .
$$

The smaller is the value of $f_{W S}^{w^{D M}}(s)$, the better solution $s$ is. Furthermore, we considered cWFG4-5 benchmarks modelled with Equations (18)-(20). To find the (Pareto) optimal solution minimizing a particular $f_{W S}^{w^{D M}}$, we solved the following equation:

$$
\nabla \sum_{i=1, \ldots, M} w_{i}^{D M} f_{i}=\overrightarrow{0}
$$

which implies that:

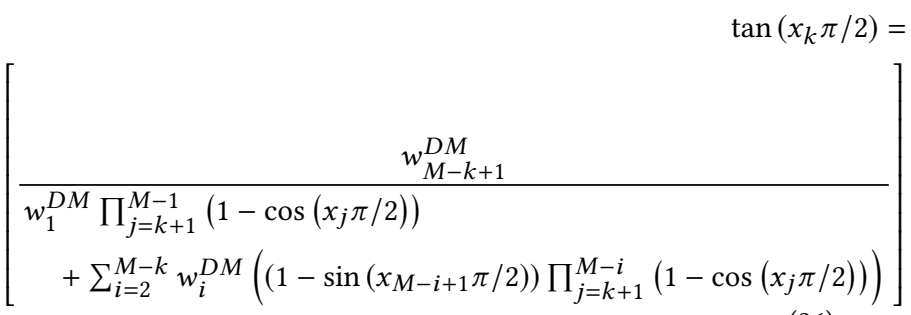

Equation (26) was iteratively solved for $k=M-1, \ldots, 1$. To find the subsequent values of $x_{k}$, we used arctan function. Precisely:

$$
x_{k}=2 \arctan (R) / \pi \text {, }
$$

where $R$ is the right side of Equation (26). In Figure 6, we illustrate the example optimal solutions for $M=2$ and 11 uniformly distributed weight vectors $w^{D M}$.

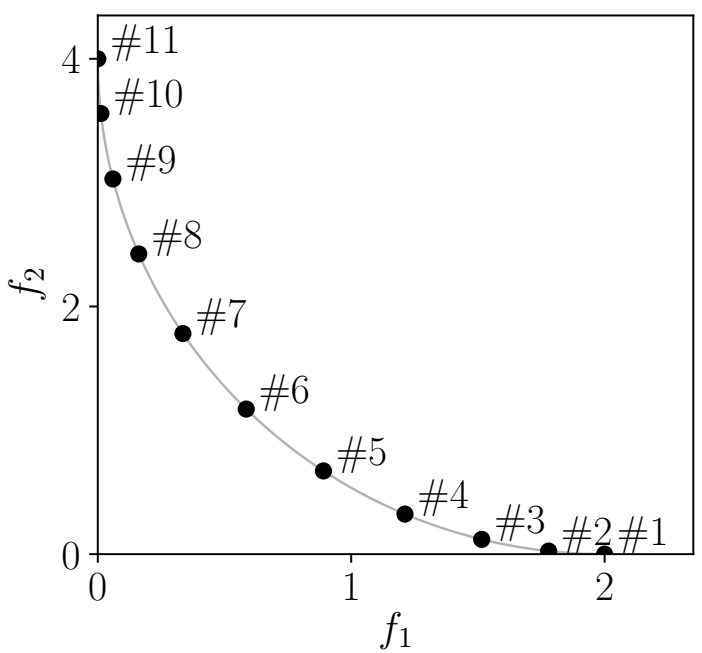

Figure 6: The PFs of cWFG4-9 (grey line) and 11 solutions minimizing $f_{W S}^{w^{D M}, i}$ (black dots). The \#i solution corresponds to $w^{D M, i}=[(i-1) / 10,1-(i-1) / 10]$ objective weight vector. 


\section{FREQUENCY OF UPDATES OF THE UTOPIAN AND NADIR POINTS}

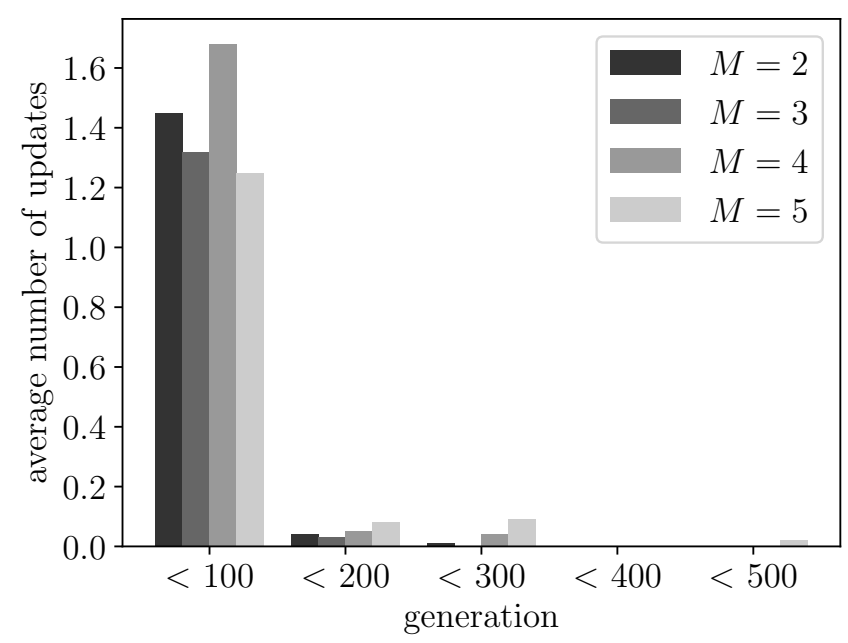

Figure 7: The average number of updates of the extreme points performed by IEMO/I in between every 100 subsequent generations.

In this section, we verify how often IEMO/I updates the utopian and a nadir points, in the course of optimization. For this reason, we applied IEMO/I to WFG3 with $M=2, \ldots, 5$ objectives and verified, for each generation, if one of these points has been updated or not. In Figure 7, we illustrate the total number of updates which have been performed within each interval of 100 subsequent generations. We wish to remind that these are averaged results, derived from 100 independent runs. Figure 7 reveals that IEMO/I updates the extreme points rarely, mostly at the beginning of the optimization. These results are not surprising because interactive EMOAs, instead of approximating an entire PF, progressively focus populations around the DM's highly preferred region in the objective space. In this perspective, in contrast to a posteriori methods, the nadir and utopian points - once set - may never be updated again during the evolutionary search. Note that both points are always estimated for the first time in the first generation. Hence, the results derived for " $<100$ " cannot be lesser than 1.0.

\section{COMPARISON OF TWO VARIANTS OF IEMO/I ASSUMING EITHER ESTIMATED OR FIXED EXTREME POINTS}

In the main paper, we assumed that the utopian and nadir points are estimated during the evolutionary search. In this section, we in vestigate whether incorporation of fixed, i.e., specified beforehand extreme points can positively affect the performance of an interactive EMOA. For this reason, we applied the original IEMO/I and and its counterpart with fixed extreme points, to WFG3 and WFG4 benchmarks with $M=2, \ldots, 5$ objectives. When it comes to fixed utopian and nadir points, these were set according to the problem's
Table 1: Averaged ARSD for the populations constructed by IEMO/I assuming either estimated or fixed extreme points, applied to WFG3 and WFG4 with $M=2, \ldots, 5(\rho=2)$.

\begin{tabular}{|c|ccc|ccc|}
\cline { 2 - 7 } \multicolumn{1}{c|}{} & \multicolumn{3}{c|}{ Estimated points } & \multicolumn{3}{c|}{ Fixed points } \\
\cline { 2 - 7 } \multicolumn{1}{c|}{} & Mean & StD & $\bar{R}$ & Mean & StD & $\bar{R}$ \\
\hline$M$ & \multicolumn{6}{c|}{ WFG3 } \\
\hline 2 & 0.77 & 0.64 & 1.47 & 0.73 & 0.47 & 1.53 \\
3 & 1.37 & 0.74 & 1.52 & 1.36 & 0.83 & 1.48 \\
4 & 2.26 & 1.23 & 1.53 & 2.45 & 1.37 & 1.47 \\
5 & 3.04 & 1.35 & 1.50 & 3.23 & 1.60 & 1.50 \\
\hline$M$ & \multicolumn{6}{c|}{ WFG4 } \\
\hline 2 & 0.60 & 1.21 & 1.51 & 0.47 & 0.79 & 1.49 \\
3 & 2.53 & 2.04 & 1.48 & 3.08 & 2.70 & 1.52 \\
4 & 3.97 & 3.16 & 1.47 & 4.02 & 2.77 & 1.53 \\
5 & 4.81 & 2.54 & 1.49 & 5.11 & 2.73 & 1.51 \\
\hline
\end{tabular}

objective ranges. Specifically, the utopian point was equal to zero vector, while the nadir point was set to $n=[2,4,6, \ldots, 2 M]$.

In Table 1, we provide ARSD attained by final populations constructed by both variants of IEMO/I. The results reveal no significant difference in the performances of these methods. This can be explained twofold. Firstly, let us observe that a fine approximation of the utopian and nadir points is not necessarily required when it comes to converging towards the DM's highly preferred region in the PF. As long as this region is in between the estimated utopian and nadir points, the method may still converge towards the optimal solution by means of a progressive search space reduction. Secondly, according to the results provided in Section 2, both utopian and nadir points are not updated often by interactive EMOAs. This mostly happens when only few DM's decision examples are provided and thus the method must explore larger regions in the objective space. The more decision examples are provided, the smaller becomes the preferred region and, consequently, the extreme solutions are not updated. For the two above reasons, similar performance of both variants of IEMO/I is not surprising as the role of the estimated extreme points is diminished and these points remain unchanged for the vast majority of generations.

\section{REFERENCES}

[1] S. Huband, P. Hingston, L. Barone, and L. While. 2006. A review of multiobjective test problems and a scalable test problem toolkit. IEEE Transactions on Evolutionary Computation 10, 5 (2006), 477-506.

[2] M. Kadziński and R. Słowiński. 2012. Interactive Robust Cone Contraction Method For Multiple Objective Optimization Problems. International fournal of Information Technology \& Decision Making 11, 02 (2012), 327-357. 